[0212-7199 (2005) 22: 2; pp 69-75] ANALES DE MEDICINA INTERNA Copyright (C) 2005 ARAN EDICIONES, S.L.

AN. MED. INTERNA (Madrid) Vol. 22, N. ${ }^{\circ} 2$, pp. 69-75, 2005

\title{
Interacciones entre medicamentos prescritos al alta en un Servicio de Medicina Interna
}

\author{
P. LÓPEZ VÁZQUEZ, C. RODRÍGUEZ MORENO, C. DURÁN PARRONDO ${ }^{1}$, F. TATO \\ HERRERO, I. RODRÍGUEZ LÓPEZ ${ }^{2}$, F. L. LADO LADO ${ }^{2}$
}

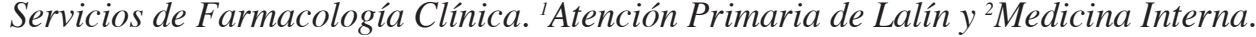
Hospital Clínico. Santiago de Compostela. A Coruña

DRUG INTERACTIONS IN THE PRESCRIPTION OF MEDICAL PATIENTS AT HOSPITAL DISCHARGE

\section{RESUMEN}

Objetivos: Estimar la frecuencia y valorar la relevancia teórica y repercusión clínica real de las interacciones medicamentosas en pacientes dados de alta desde un área médica.

Material y métodos: Se revisan los tratamientos al alta de 412 pacientes de forma aleatoria. Se identifican las potenciales interacciones usando la Guía de Terapia Farmacológica Medimecum. Para la clasificación según su relevancia clínica teórica se usa la propuesta por Hansten y Horn.

Resultados: Se encuentran 329 interacciones teóricas. El 39,9\% de los pacientes presentaban al menos una interacción [IC 95\%, 34,9\%$44,9 \%$ ]. El 52,6\% de las interacciones eran clase 3 según Hansten, aquellas en las que ha de tomarse alguna medida para disminuir el riesgo de efectos indeseables. No se encontró ninguna interacción clase 1 ó 2 de Hansten, categorías de mayor gravedad. Las interacciones clase 3 más frecuentes implican a anticoagulantes orales y digoxina. Las medidas de control fueron realizadas en el $100 \%$ de los casos para las interacciones entre anticoagulantes orales y otros fármacos. En las interacciones de digoxina y otros fármacos las medidas de control recomendadas no fueron realizadas suficientemente. Un paciente reingresó con niveles por encima de rango de digoxina, habiendo sido dado de alta con dos potenciales interacciones.

Conclusiones: La frecuencia de interacciones en pacientes dados de alta desde el área médica es alta. La relevancia teórica y la práctica parece ser baja.

PALABRAS CLAVE: Interacciones farmacológicas. Estudios de utilización de medicamentos. Prescripción.

\section{ABSTRACT}

Objectives: To detect and evaluate the clinical significance of drugs interactions in patients discharged from hospital.

Material and methods: We retrospectively screened the medication for potential drug interactions of 412 patients discharged. Interactions were catalogued according to clinical importance following the Hansten and Horn's classification.

Results: Three hundred twenty-nine potential interactions were detected. The $39.9 \%$ of the patients had at least one potentially interacting drug combination. The $52.6 \%$ of the interactions were catalogued as Class 3 , bearing in mind minimizing the risk of the interaction. We did not find any Class 1 or 2 interactions, which have potentially major severity. Oral anticoagulants and digoxin were the most frequently implicated drugs. The patient monitoring was well done in the $100 \%$ of the interactions of Oral anticoagulants with other drug, but in the interactions of digoxin with another, this control was not done adequately. One patient was re-hospitalised due to high levels of digoxin, he had been discharged with two potential interactions.

Conclusions: The frequently of potential drug interactions in medical patients at hospital discharged was high, but the clinical significance appear to be low.

KEY WORDS: Drug Interactions. Prescriptions. Drug utilization studies.

López Vázquez P, Rodríguez Moreno C, Durán Parrondo C, Tato Herrero F, Rodríguez López I, Luis Lado Lado F. Interacciones entre medicamentos prescritos al alta en un servicio de Medicina Interna. An Med Interna (Madrid) 2005; 22: 69-75.

\section{INTRODUCCIÓN}

Entendemos como interacción medicamentosa la acción que un medicamento ejerce sobre otro, de modo que éste experimenta un cambio cualitativo y cuantitativo en sus efectos (1). La importancia sanitaria teórica de esta situación es grande pues el resultado de una interacción puede suponer bien el fracaso terapéutico o bien la aparición de efectos adversos; ambas posibilidades ponen en riesgo al paciente y añaden otros perjuicios sanitarios como una mayor complejidad del diagnóstico y tratamiento, la hospitalización del paciente o la prolongación de la estancia hospitalaria. Por ello es de gran importancia conocer, en la práctica clínica cotidiana, la frecuencia con que se producen estas interacciones y la repercusión que tienen sobre la salud de los enfermos y sobre la utilización de los servicios sanitarios. Además no hay que

Trabajo aceptado: 10 de noviembre de 2004

Correspondencia: Carlos Rodríguez Moreno. Servicio de Farmacología Clínica. Hospital Clínico Universitario de Santiago. Travesia Choupana, s/n. 15706 Santiago de Compostela (A Coruña). e-mail: carlos.rodriguez.moreno@ sergas.es 
olvidar que, en bastantes ocasiones, las interacciones son potencialmente evitables.

Se ha realizado un estudio de utilización de medicamentos con los siguientes objetivos: a) estimar la prevalencia de interacciones medicamentosas en pacientes dados de alta desde un Servicio de Medicina Interna; b) estimar la relevancia teórica de las mismas; c) en el caso de las interacciones clasificadas teóricamente como relevantes, determinar la repercusión clínica; y d) abordar estrategias encaminadas a aumentar la percepción del clínico sobre el riesgo que comportan estas interacciones y las medidas que podrían tomarse para minimizarlo.

\section{MATERIAL Y MÉTODOS}

Se trata de un estudio transversal cuya población de estudio la constituyen todos los pacientes dados de alta desde el Servicio de Medicina Interna del Hospital Clínico Universitario de Santiago de Compostela durante el año 2003 (1.117 pacientes). Se seleccionó una muestra aleatoria de dicha población ( $\mathrm{n}=412$ pacientes), procediéndose a la revisión de los informes de alta, de donde se recogen los siguientes datos: sexo, edad, fecha de alta, diagnósticos al alta (los 6 primeros), y todos los medicamentos prescritos al alta.

Para la identificación de las interacciones medicamentosas potenciales se utilizó la tabla de interacciones de la guía de terapia farmacológica Medimecum 2003 (2), por ser una fuente de información ampliamente utilizada por el clínico en nuestro medio, de contrastada calidad, de fácil manejo y a la que de forma rápida y eficiente se podría recurrir para evitar interacciones indeseables.

Para el segundo objetivo, la clasificación de las interacciones medicamentosas según su relevancia teórica, hemos utilizado la propuesta de Hansten y Horn (3), un texto clásico considerado internacionalmente de referencia, que las distribuye en cinco clases: 1) evitar la combinación (el riesgo supera el beneficio); 2) combinación permitida sólo en especiales circunstancias; 3) la combinación de los fármacos precisa usar distintos mecanismos para minimizar el riesgo. Las clases 4 y 5 serían aquellas en las que el potencial riesgo para el paciente es pequeño o existen datos a favor de que no existe interacción.

Para el tercer objetivo (determinar la repercusión clínica real de estas interacciones), y debido al carácter retrospectivo del estudio que limitó el tipo de medidas de seguimiento y valoración, se revisaron las historias clínicas de los pacientes con interacción para detectar posibles acontecimientos relacionados con la misma, incluyendo la existencia de reingresos con un diagnóstico relacionado con la interacción en los tres meses posteriores a su alta hospitalaria. En las interacciones clasificadas en la clase 3, es decir, aquellas en que el peligro de la combinación disminuye si se lleva a cabo alguna medida de control, se revisaron las historias de los pacientes afectados con el fin de determinar si estas medidas fueron realizadas. Una vez conocida la naturaleza de las interacciones tipo 3, las medidas de control que comprobamos fueron: controles del INR y ajuste de la dosis de anticoagulante oral (ACO), determinaciones de parámetros bioquímicos de laboratorio y determinaciones de niveles plasmáticos de fármacos.

Se procedió al análisis descriptivo de la muestra con el paquete estadístico SPSS 10.0. Los resultados son expresados como proporción o media junto con su desviación estándar. Se realizó comparación de medias en muestras independientes con la prueba t de Student con un nivel de significación estadística de $\mathrm{p}<0,05$.

\section{RESULTADOS}

\section{GENERAL DE LA MUESTRA}

Se revisaron un total de 412 informes de alta de pacientes. En la tabla I se detallan los datos demográficos y la prescripción de medicamentos en esta muestra. El 58,3\% de los pacientes incluidos en el estudio son varones y el $41,5 \%$ mujeres. La edad media fue de $69 \pm 17$ años, con un rango comprendido entre 15 y 106 años. Fueron prescritos al alta un total de 2.062 medicamentos, que corresponde a una media de 5 medicamentos por paciente (rango entre 0 y 12).

En la tabla II se muestran los medicamentos mas prescritos agrupados según la clasificación anatómico terapéutica (ATC). Los medicamentos más prescritos fueron omeprazol (25,9\% de las prescripciones), ácido acetil salicílico (20,6\%), furosemida $(19,6 \%)$, digoxina $(19,4 \%)$, nitroglicerina transdérmica $(16,7 \%)$. En la tabla III se detalla la distribución de los diagnósticos de la muestra. Las patologías más frecuentes incluidas como primer diagnóstico son insuficiencia cardiaca (supone el 8\% del total), infección respiratoria (6\%), neumonía adquirida en al comunidad $(6 \%)$, enfermedad pulmonar obstructiva crónica reagudizada $(5,6 \%)$. Si se consideran el global de los diagnósticos de todos los pacientes, no sólo el primer diagnóstico dado al alta, las dos patologías más frecuentes coinciden con la distribución anterior.

\section{TABLA I}

CARACTERÍSTICAS DE LOS PACIENTES INCLUIDOS EN EL ESTUDIO

\begin{tabular}{|c|c|c|c|}
\hline & & $\begin{array}{c}\text { General de la } \\
\text { muestra (\%) } \\
n=412\end{array}$ & $\begin{array}{c}\text { Población con } \\
\text { interacción(\%) } \\
n=147\end{array}$ \\
\hline Sexo* & $\begin{array}{l}\text { Masculino } \\
\text { Femenino }\end{array}$ & $\begin{array}{l}240(58,4) \\
171(41,6)\end{array}$ & $\begin{array}{l}86(58,5) \\
61(41,5)\end{array}$ \\
\hline Edad* & $\begin{array}{l}\text { Hasta } 30 \text { años } \\
\text { De } 31 \text { a } 64 \\
\text { De } 65 \text { a } 74 \\
75 \text { o más }\end{array}$ & $\begin{array}{c}13(3,2) \\
114(27,7) \\
87(21,2) \\
197(47,9)\end{array}$ & $\begin{array}{c}2(1,4) \\
25(17) \\
31(21,1) \\
89(60,5)\end{array}$ \\
\hline $\begin{array}{l}\text { Número de } \\
\text { medicamentos }\end{array}$ & $\begin{array}{l}0 \\
1 \text { ó } 2 \\
\text { De } 3 \text { a } 6 \\
\text { De } 7 \text { a } 10 \\
11 \text { o más }\end{array}$ & $\begin{array}{c}12(2,9) \\
72(17,5) \\
202(49) \\
109(26,5) \\
17(4,1)\end{array}$ & $\begin{array}{c}0 \\
2(1,4) \\
59(40,1) \\
75(51) \\
11(7,5)\end{array}$ \\
\hline
\end{tabular}

* datos perdidos para un paciente

\section{DE LA POBLACIÓN CON INTERACCIÓN}

Se encuentran un total de 329 interacciones teóricas, que aparecen en 147 pacientes, lo que supone un 39,9\% [IC 95\% 


\begin{tabular}{|c|c|}
\hline \multicolumn{2}{|l|}{ TABLA II } \\
\hline \multicolumn{2}{|c|}{$\begin{array}{c}\text { PRESCRIPCIÓN POR GRUPOS SEGÚN CLASIFICACIÓN } \\
\text { ANATÓMICO TERAPÉUTICA }\end{array}$} \\
\hline Grupos terapéuticos & $\begin{array}{c}\text { Número de pacientes } \\
(\%)\end{array}$ \\
\hline Inhibidores de la bomba de protones (A02B2) & $167(45,5)$ \\
\hline Diuréticos de asa (CO3B) & $148(35,9)$ \\
\hline Antiagregantes plaquetarios (B01B) & $136(33)$ \\
\hline B-agonistas selectivos (R03A1A) & $110(26,7)$ \\
\hline Ansiolíticos, sedantes e hipnóticos (N05B) & $89(21,6)$ \\
\hline Nitratos solos (C01D1A) & $84(20,4)$ \\
\hline Digitálicos (C01A1) & $80(19,4)$ \\
\hline Inhibidores de la ECA (CO2E1) & $65(15,8)$ \\
\hline Corticoides por inhalación (R03A1D) & $64(15,5)$ \\
\hline Corticoides solos (H02A) & $60(14,6)$ \\
\hline Anticolinérgicos (R03A1D) & $54(13,1)$ \\
\hline Antagonistas del calcio (C01D2) & $52(12,6)$ \\
\hline Anticoagulantes orales (B01A1) & $51(12,4)$ \\
\hline $\begin{array}{l}\text { Bloqueantes de los receptores AT1 de } \\
\text { angiotensina II (CO2E2) }\end{array}$ & $51(12,4)$ \\
\hline Diuréticos ahorradores de potasio (CO3C) & $48(11,6)$ \\
\hline Antipsicóticos solos (N05A1) & $40(9,7)$ \\
\hline
\end{tabular}

$34,9 \%-44,9 \%$ ] de todos los pacientes de nuestra muestra susceptibles de sufrir al mínimo una interacción (aquellos que reciben 2 o más medicamentos, $\mathrm{n}=368$ ).

En la tabla I se puede observar que la distribución por edades en la muestra y en la población con interacción es diferente. La edad media de los pacientes con al menos una interacción en su tratamiento al alta en este caso aumenta a 73,1 \pm 14,6 años y el número medio de medicamentos pasa a ser 6,92 $\pm 2,26$ y es significativamente mayor que en el grupo de pacientes sin interacción $(6,92$ vs. 3,94, $\mathrm{p}<0,001)$. El número medio de interacciones por paciente es de $2,25 \pm 1,54$, con un rango de 1 a 7 interacciones por paciente. El 80,5\% de los casos tenían 2 o más interacciones al alta. Veintiuno de los pacientes incluidos en nuestro estudio tenían 7 potenciales interacciones en su tratamiento.

Las enfermedades mas frecuentes incluidas como primer
TABLA III

\section{DIAGNÓSTICOS MÁS FRECUENTES}

\begin{tabular}{lc}
\hline Diagnóstico & $\begin{array}{c}\text { Número de pacientes } \\
(\%)\end{array}$ \\
\hline Insuficiencia cardiaca & $89(21)$ \\
Infección respiratoria & $73(17,7)$ \\
EPOC* & $72(17,5)$ \\
Hipertensión arterial & $68(16,5)$ \\
Neumonía (comunitaria o nosocomial) & $67(16,4)$ \\
Diabetes mellitus & $53(12,8)$ \\
Fibrilación auricular & $45(10,9)$ \\
Infección vías urinarias & $35(8,5)$ \\
Cardiopatía isquémica & $31(7,5)$ \\
Neoplasia de órgano sólido & $25(6)$ \\
VIH & $20(4,8)$ \\
Sepsis & $16(3,8)$ \\
Síndrome de privación alcohólica & $15(3,6)$ \\
\hline
\end{tabular}

${ }^{*}$ enfermedad pulmonar obstructiva crónica

TABLA IV

GRUPOS SEGÚN CLASIFICACIÓN ANATÓMICO TERAPÉUTICA E INTERACCIÓN

\begin{tabular}{lc}
\hline Grupos terapéuticos & Número de interacciones \\
\hline Digitálicos (C01A1) & 152 \\
Diuréticos de asa (CO3B) & 84 \\
Inhibidores de la bomba de protones (A02B2) & 75 \\
Sulfonilureas (A10B1A) & 52 \\
Anticoagulantes orales (B01A1) & 49 \\
Antagonistas del calcio (C01D2) & 29 \\
Corticoides solos (H02A) & 27 \\
Ansiolíticos, sedantes e hipnóticos (N05B) & 24 \\
\hline
\end{tabular}

diagnóstico fueron: IC (17\%), EPOC reagudizado $(7,6 \%)$, neumonía $(4,6 \%)$.

Los medicamentos que más frecuentemente estuvieron implicados fueron digoxina (46,3\%), omeprazol (18,6\%), ace-

TABLA V

LISTA DE LAS INTERACCIONES INCLUIDAS EN EL GRUPO 3 SEGÚN HANSTEN

\begin{tabular}{llc}
\hline Interacciones & Efecto adverso potencial & Número de interacciones (\%) \\
\hline Digoxina-diuréticos de asa & Incrementa riesgo de toxicidad digitálica por hipokalemia & $59(33,3)$ \\
Anticoagulantes-otros medicamentos & Disminución o aumento del efecto anticoagulante & $49(27,7)$ \\
Digoxina - IBP & Incremento de niveles séricos de digoxina & $36(20,3)$ \\
Digoxina - espironolactona & Incremento de niveles séricos de digoxina & $14(7,9)$ \\
Captopril- diuréticos de asa & Hipotensión & $5(2,8)$ \\
Digoxina - telmisartán & Incremento de niveles séricos de digoxina (principalmente niveles valle) & $3(1,7)$ \\
Digoxina - diltiazem & Incremento de niveles séricos de digoxina & $3(1,7)$ \\
Valproico- isoniazida & Incremento de niveles séricos de valproico & $2(1,1)$ \\
Digoxina -amiodarona & Incremento de niveles séricos de digoxina & $2(1,1)$ \\
Clonazepam - ácido valproico & Crisis de ausencia & $1(0,7)$ \\
Metadona - ritonavir & Disminución del efecto de la metadona & $1(0,57)$ \\
Prednisolona - isoniazida & Reducción de las concentraciones plasmáticas de prednisolona & $1(0,57)$ \\
Indinavir - ritonavir & Aumento de las concentraciones plasmáticas de indinavir & $1(0,57)$ \\
\hline
\end{tabular}


nocumarol $(14,9 \%)$ y furosemida $(13,7 \%)$. En la tabla IV se muestran agrupados en las categorías de la clasificación anatómica terapéutica (ATC).

En cuanto a la relevancia de estas interacciones, no se encontró ninguna interacción incluida en las clases 1 y 2 de la clasificación de Hansten y Horn (3). Se encontraron 177 interacciones de clase 3 que suponen el 53,8\% del total, afectando a 103 de los pacientes incluidos en el estudio $(27,9 \%)$ y que se recogen en la tabla $\mathrm{V}$.

El resto de las interacciones detectadas por el primer método (siguiendo la clasificación del Medimecum) fueron incluidas dentro de la clase 4 ó 5.

Respecto a la repercusión clínica de las interacciones primero se valoró la realización de las medidas y acciones encaminadas a minimizar el impacto de las interacciones. Se encontró que para la interacción de clase 3 más frecuente (anticoagulantes (ACO) con otros medicamentos), los controles del INR se realizaron en todos los pacientes. En la siguiente por orden de frecuencia (digoxina con otros medicamentos) se encontró que sólo en el $22 \%$ de los pacientes se solicitó determinación de niveles plasmáticos durante su ingreso reduciéndose esta cifra a menos del $1 \%$ en los tres meses posteriores al alta. El control del potasio sérico se realizó en todos los pacientes, durante su ingreso, y en el $43 \%$ en los tres meses posteriores. Cuando la medida recomendada era la monitorización clínica del efecto farmacológico no se pudo verificar si esta fue llevada a cabo o no.

En cuanto a reingresos secundarios a las interacciones farmacológicas se halló que uno de los pacientes reingresó al mes de ser dado de alta con niveles de digoxina por encima del rango $(3,3 \mathrm{ng} / \mathrm{ml})$ presentando dos potenciales interacciones con digoxina: digoxina-inhibidores de la bomba de protones, y digoxina-furosemida. El paciente ingresó con el diagnóstico de "insuficiencia cardiaca". Presentaba también cifras de creatinina elevadas $(4,7 \mathrm{mg} / \mathrm{dl})$ y aunque los niveles de digoxina encontrados pudieron deberse total o parcialmente a la insuficiencia renal no se puede descartar la participación de las interacciones descritas.

En cuanto al cuarto objetivo, se realizó una sesión informativa conjunta con el Servicio de Medicina Interna, donde se expusieron los resultados de este estudio, así como las implicaciones que se detallan en la discusión, y se propusieron medidas y actitudes que podrían mejorar la situación encontrada. En esta sesión se repartió un material informativo, elaborado por nuestro servicio, sobre las interacciones entre medicamentos, su relevancia clínica y su prevención.

\section{DISCUSIÓN}

\section{DEL MÉTODO COMPARANDO CON OTROS ESTUDIOS}

Con el presente estudio se pretendió valorar tanto cuantitativamente como cualitativamente la prevalencia de las interacciones medicamentosas en los tratamientos con los que pacientes son dados de alta tras su ingreso en el área médica.

En la literatura encontramos otros estudios transversales sobre interacciones farmacológicas en diferentes ámbitos, tanto hospitalario como extrahospitalario (4-9). Dentro del primero algunos son realizados en Unidades de Críticos $(10,11)$ justificándolos porque los pacientes que están en dichas unidades suelen recibir un gran número de medicamentos simul- táneamente, en Unidades de Urgencias (12-15), y otros en unidades del área médica (16-18) similares al que se ha realizado.

Se ha elegido un Servicio de Medicina Interna para la realización de este estudio, por las características de los pacientes que ingresan en él, generalmente de edad avanzada y con pluripatología, lo que conlleva un número elevado de medicamentos prescritos y una respuesta modificada a éstos (múltiples modificaciones cinéticas -flujo sanguíneo renal, porcentaje de agua y grasa corporal, motilidad intestinal, etc. y farmacodinámicas -capacidad homeostática, densidad de receptores, etc.), situación que favorece las interacciones medicamentosas y agrava sus consecuencias (19).

Se ha tomado como referencia los medicamentos prescritos al alta primero porque suelen coincidir con los administrados durante el ingreso, y segundo, porque tras ser dado de alta el paciente deja un medio estrechamente controlado y vigilado, como es el hospitalario donde es fácil detectar los problemas con prontitud y tomar las medidas necesarias para corregirlos y pasa al medio ambulatorio, donde no existen facilidades para realizar ese control y podrían pasar desapercibidos.

Se ha optado por un estudio retrospectivo porque el acceso a las historias de Medicina Interna por parte de nuestro servicio implica una notificación y acuerdo con ellos y, este conocimiento, podría afectar los resultados en caso de un estudio prospectivo; nos parecía interesante evaluar el problema en situación real y cotidiana sin el sesgo de saberse evaluados.

Respecto a la detección de las interacciones y posterior valoración de la relevancia había diferentes posibilidades. El documento descriptivo de interacciones más ampliamente utilizado para estudios con esta metodología en nuestro país es el Hansten y Horn (3). Es además, el texto que más específicamente se orienta a clasificar las interacciones por su relevancia. En los estudios no españoles con esta metodología la fuente mas utilizada es el Drug-Reax (Micromedex) (20) que es una fuente exhaustiva pero quizá algo menos orientada hacia la relevancia de la interacción. La utilización del Medimecum como texto para la detección de interacciones, se explica por las pretensiones de nuestro estudio. Como se desprende de los objetivos, se pretendía, además de una valoración de un aspecto concreto de la prescripción de medicamentos, realizar una cierta labor docente o "cultural" en el sentido de difundir entre los clínicos involucrados una mayor conciencia hacia la necesidad de una prescripción reflexiva, documentada y cuidadosa. El texto mas accesible, cómodo y difundido en los pasillos de nuestro hospital es el Medimecum por lo que las interacciones descritas en este texto reflejan mejor las interacciones que un clínico puede detectar y evitar en el momento de la prescripción. Para valorar posteriormente la relevancia de las interacciones detectadas, la opción obvia es el Hansten y Horn (3), el único texto que además de orientado a la relevancia de las interacciones establece una clasificación en categorías de relevancia que facilita su aplicación a este estudio.

Para valorar del impacto real de las interacciones, lo ideal hubiera sido un seguimiento estrecho de los pacientes y realización de una encuesta telefónica, postal o personal al enfermo y a su médico de referencia. Al ser un estudio retrospectivo lo único posible era una búsqueda también retrospectiva en dos vertientes: a) modificación de las actuaciones médicas que implicaba la interacción (determinaciones analíticas, ajus- 
tes de dosis, etc.); y b) acontecimientos relacionados con la interacción lo suficientemente importantes para que consten en la historia (reingresos, determinaciones analíticas, etc.).

Por último, y en la línea comentada mas arriba, las intenciones docentes o culturales de este estudio se abordaron de forma puntual con un información escrita y presencial que puede enmarcarse en una relación mas a largo plazo, ya existente, de formación continuada entre ambos servicios donde se incluye la atención de consultas terapéuticas, sesiones conjuntas, etc.

\section{DE LOS DATOS COMPARANDO CON OTROS ESTUDIOS}

La frecuencia de interacciones encontrada en nuestro trabajo (39,9\% de los pacientes), se encuentra dentro del amplio margen detectado en los estudios publicados, que puede ser tan baja como una incidencia de 1,9 por 1.000 pacientes-año en un estudio realizado en un área de salud de Inglaterra sobre pacientes ambulatorios (21), o tan alta como el $60 \%$ encontrado en un estudio sobre pacientes dados de alta en un Hospital en Suiza (18). Esta amplia diferencia se debe a factores como son la variabilidad en la procedencia de la muestra estudiada (población general, centros de salud o hospitales), las diferentes fuentes de información utilizadas como guías para definir las interacciones o la diferente rigurosidad a la hora de definir una interacción.

Si comparamos la frecuencia de interacciones hallada con los datos de otros estudios publicados y cuyo ámbito sea el área médica de un hospital encontramos cifras ligeramente más altas que la nuestra. Egger y cols. (18) publicaron en el 2003 un estudio retrospectivo que contaba con un total de 500 pacientes dados de alta desde una Unidad del área médica, hallando que el $60 \%$ [IC 95\% 55,7\%-64,3\%] de los pacientes estudiados tenían al menos una interacción. Porcentajes similares encuentran Gepper y cols. (16) con un 66,9\% de los pacientes con al menos una interacción al alta, y Kohler y cols. (17) con un $60 \%$. Una posible explicación de esta diferencia podría ser el haber usado una fuente bibliográfica inicial para la detección de las interacciones diferente a la que se usó en los estudios anteriormente mencionados (Medimecum frente a la base de datos Drug-Reax de Micromedex).

El hecho de usar diferentes fuentes afecta también a la clasificación de las interacciones según su relevancia clínica por lo que no podemos hacer una comparación directa entre nuestros resultados y los de otros estudios. Por ejemplo, en Egger y cols. la interacción entre Amiodarona-Metoprolol es clasificada dentro del grupo de mayor severidad, mientras que según la clasificación seguida por nosotros sería de clase 3. Según la clasificación propuesta por Hansten y Horn (3), una combinación de medicamentos es incluida en el grupo de mayor severidad no sólo dependiendo de la gravedad del potencial efecto indeseable que pueda causar sino también dependiendo de si existe o no alguna medida para minimizar este riesgo. En caso de que exista esta medida la combinación deja de estar considerada como de alto riesgo.

Debido a la metodología empleada es posible que se haya infraestimado el número de interacciones relevantes: por ejemplo, para la interacción entre los IECA con diuréticos de asa los textos suelen incluir uno o dos IECA, cuando sabemos que esto podría ser generalizado para todos los IECA. Y también, hemos encontrado interacciones no recogidas como rele- vantes por Hansten y Horn (3) pero que, pueden considerarse claramente de riesgo como IECA con ahorradores de potasio (22).

En cuanto a la repercusión real de las interacciones medicamentosas (en general en todos los trabajos se valoran rehospitalizaciones de los pacientes implicados), parece ser escasa. En nuestro estudio, aunque sólo se haya encontrado un paciente con un posible ingreso relacionado con una interacción medicamentosa, no se puede concluir que las consecuencias derivadas de las mismas sean mínimas pues pudieron haber ocurrido efectos indeseables secundarios a las potenciales interacciones medicamentosas que no precisaran hospitalización pero sí atención médica y por tanto no pudieron ser detectados.

Una constante en los resultados de todos los trabajos publicados sobre interacciones, y que tiene una lógica aplastante, es que el número de medicamentos es mayor en el subgrupo con interacción que en el subgrupo que no tiene interacción $(10,17,18)$ lo que también se confirma en nuestro estudio.

Llama especialmente la atención, no sólo en este estudio sino también en los revisados, que los fármacos más frecuentemente implicados son en principio ampliamente conocidos por el médico como también suponemos que son las interacciones en las que intervienen. Creemos por tanto que los riesgos de la administración conjunta de los mismos son asumidos por el médico, y también tenemos en cuenta que pueden haber tomado medidas complementarias (en el caso de furosemida-digoxina administrar simultáneamente un diurético ahorrador de potasio o suplementos de potasio) que por las limitaciones que tiene el diseño de nuestro estudio no han sido tenidas en cuenta. Aun así el hecho de tomar estas medidas no haría desaparecer el riesgo por lo que igualmente sería necesario la monitorización del paciente ambulatoriamente.

\section{DE LA INTERPRETACIÓN DE LOS RESULTADOS DE NUESTRO ESTUDIO}

Este estudio nos sugiere que los clínicos del Servicio de Medicina Interna de nuestro hospital conocen bastante bien las posibles interacciones que conllevan riesgos importantes para los pacientes porque no hemos encontrado ninguna de las clases 1 y 2 según la clasificación de Hasten y Horn (3). En cuanto a su actitud ante interacciones posiblemente relevantes y que necesitan una intervención para asegurar una correcta relación beneficio/riesgo de su prescripción nos encontramos ante dos extremos: La más frecuente, los ACO con otros medicamentos y que implica una monitorización del INR es realizada en todos los casos, muy probablemente porque forma parte del control rutinario de los pacientes en tratamiento con acenocumarol; la corriente actual en este tema es intentar utilizar los medicamentos que sean más seguros (es decir, que menos interaccionen con ACO); ahora bien, si el paciente requiere un medicamento que interacciona (por ejemplo una quinolona), se utiliza y se adelanta el control de INR ajustando la dosis de ACO (23).

Sin embargo, en el caso de las interacciones con digoxina consideramos que la práctica es mejorable. El número de pacientes de nuestro estudio que tenían una asociación con digoxina considerada de riesgo es alta y tan sólo en un pequeño porcentaje se han determinado los niveles plasmáticos tanto antes como después del alta hospitalaria. 
Como se ha demostrado $(24,25)$ que tanto a nivel de Atención Primaria como de Urgencias en España los pacientes en tratamiento con digoxina presentan niveles infraterapéuticos de este fármaco (hasta un $57 \%$ de los pacientes estudiados) asociados a infradosificación, se nos ocurre pensar que quizá ambos hechos puedan estar relacionados. Debido al conocimiento de que el tratamiento con digoxina implica un riesgo importante de intoxicación digitálica la respuesta instintiva del clínico sería infradosificar con lo que evita el riesgo de intoxicación. Actitud que empieza a parecer razonable tras las últimas recomendaciones extraídas de los análisis "post-hoc" del estudio del Digitalis Investigation Group (DIG) (26) para el tratamiento de la insuficiencia cardiaca, que demostró disminución en la mortalidad en el subgrupo de pacientes (varones) con niveles entre 0,5-0,8 ng/ml. En la sesión informativa recalcamos a nuestros clínicos que la única medida que garantiza mantener el beneficio terapéutico de la digoxina y a su vez evitar el riesgo de intoxicación sería una monitorización suficiente de sus niveles plasmáticos.

Otra situación que nos parece que se resuelve engañosamente bien, son las interacciones que involucran a diuréticos de asa y que implican un riesgo de hipotasemia. En nuestro estudio durante la estancia hospitalaria se determina el potasio sérico a todos los pacientes, hecho que forma parte de la rutina, pero una vez dados de alta tan sólo se realiza esta determinación en el $43 \%$ de los pacientes. Suponemos que la tendencia es asociar un diurético ahorrador de potasio o suplementos de potasio pero, en nuestra opinión esto no garantiza una potasemia normal y debería realizarse sistemáticamente su determinación.

Por otro lado, aunque esto no forma parte de los objetivos del estudio y por tanto no pueden ser conclusiones del mismo, como consecuencia de la revisión realizada de la distintas fuentes de información sobre interacciones hemos detectado una gran disparidad en la clasificación en grados de relevancia clínica entre ellas. En muchas ocasiones esta relevancia se basa en la existencia de evidencia bibliográfica de dicha interacción y no tanto la repercusión clínica que pueda tener. Por ello se plantea la necesidad de realizar un nuevo estudio que compare las distintas fuentes, según su capacidad para detectar y clasificar las interacciones, y quizás proponer una nueva clasificación.

Los resultados obtenidos en el presente trabajo nos permiten concluir que: a) existe una elevada frecuencia de interacciones potenciales al alta; b) ninguna de ellas pertenece a las categorías mas altas de peligrosidad o relevancia; c) tan sólo alrededor de la mitad de ellas pertenecen a la categoría de relevancia media (requiere alguna acción del clínico); y d) el cumplimiento de las medidas orientadas a minimizar el impacto de las interacciones de relevancia media es bajo.

Este último punto es el mas preocupante y pensamos que quizás las siguientes iniciativas podrían mejorar esta situación:

La instalación de un programa informático, ya en funcionamiento en otros países $(27,28)$, que detecte las interacciones medicamentosas y emita un aviso al clínico en el momento de la prescripción. Sería interesante que además de alarmar de la interacción, avise del potencial efecto adverso y de la conducta a seguir.

Concienciar al clínico de la importancia de llevar a cabo las medidas de control necesarias para minimizar el riesgo de las interacciones. Parece que sería especialmente necesario a nivel de Atención Primaria, donde generalmente se deriva el control de los pacientes de nuestro estudio.

Y por último, como queda demostrado en la mayor parte de estudios sobre interacciones, el único factor claramente relacionado con las mismas es el número de medicamentos prescritos al alta, luego una medida fundamental para reducir éstas sería usar el número mínimo indispensable de medicamentos

\section{Bibliografía}

1. De Cos MA. Interacciones de fármacos y sus implicaciones clínicas. En: Flórez J, Armijo JA, Mediavilla A, editores. Farmacología Humana, $4^{\mathrm{a}}$ edición. Barcelona: Masson, 2003, p. 175-6.

2. Esteban C, Maciá MA, Ibáñez C, Fernández Esteban I, De la Morena L. Interacciones medicamentosas. En: Villa LF, editor. Medimecum, guía de terapia farmacológica, $7^{\text {a }}$ edición. España: Addis Internacional Ltd; 2002, p. 803-38.

3. Hansten PD, Horn JR. Managing clinically important drug interactions. St Louis: Facts and Comparisons, 2003.

4. Guédon-Moreau L, Ducrocq D, Duc MF, Quieureux Y, L'Hote C, Deligne J, et al. Absolute contraindications in relation to potencial drug interactions in outpatient prescripcions: analysis of the first five million prescriptions in 1999. Eur J Clin Pharmacol 2003; 59: 689-95.

5. Recalde JM, Zunzunegui MV, Beland F. Interacciones entre medicamentos prescritos en la población mayor de 65 años. Aten Primaria 1998; 22: 434-9.

6. Calvet A, Díez M, Pérez MT, Esteras J. Interacciones farmacológicas en tratamientos crónicos: medidas correctoras para su prevención en un área básica de salud rural. Aten primaria 2001; 27: 91-6.

7. García A, García MA, Morilla J, Lobato F, Portillo J, Pérez A. Detección de interacciones medicamentosas en pacientes crónicos mediante un programa informático. Utilidad práctica en un centro de salud. Aten primaria. 1997;19: 138-41.
8. Sáenz Calvo A, Ausejo Segura M, Cruz Martos E, González Álvaro A, Pérez Cayuela P, Rosado López A. Drug-to-drug interactions and pharmaceutical advice. Aten Primaria 1996; 17: 559-63.

9. Comet D, Casajuana J, Bordas JM, Fuentes MA, Arnaiz JA, Nunez B, et al. Drug interactions in chronic prescription. Aten Primaria. 1997; 20: 71-4.

10. Sierra P, Castillo J, Gómez M, Sorribes V, Monterde J, Castano J. Potential and real drug interactions in critical care patients. Rev Esp Anestesiol Reanim 1997; 44: 383-7.

11. Dasta JF. Drug use in a surgical intensive care unit. Drug Intell Clin Pharm 1986; 20: 752-6.

12. Hohl CM, Dankoff J, Colacone A, Afilalo M. Polypharmacy, adverse drug-related events, and potential adverse drug interactions in elderly patients presenting to an emergency department. Ann Emerg Med 2001; 38: 666-71.

13. Beers MH, Storrie M, Lee G. Potential adverse drug interactions in the emergency room. An issue in the quality of care. Ann Intern Med 1990; 112: $61-4$

14. Gaddis GM, Holt TR, Woods M. Drug interactions in at-risk emergency department patients. Acad Emerg Med 2002; 9: 1162-7.

15. Heininger-Rothbucher D, Bischinger S, Ulmer H, Pechlaner C, Speer $\mathrm{G}$, Wiedermann CJ. Incidence and risk of potential adverse drug interactions in the emergency room. Resuscitation 2001; 49: 283-8. 
16. Geppert U, Beindl W, Hawranek T, Hintner H. Drug interactions in clinical practice. A pilot project for quality assurance in prescribing. Hautarzt 2003; 54: 53-7.

17. Kohler GI, Bode-Boger SM, Busse R, Hoopmann M, Welte T, Boger RH. Drug-drug interactions in medical patients: effects of in-hospital treatment and relation to multiple drug use. Int J Clin Pharmacol Ther 2000; 38: 504-13.

18. Egger SS, Drewe J, Schlienger RG. Potencial drug-drug interactions in the medication of medical patients at hospital discharge. Eur J Clin Pharmacol 2003; 58: 773-8.

19. Rosso C, Geniz MA, Serrani MI. Factores fisiológicos que condicionan la respuesta a los fármacos. En: SEFC (ed). Manual del residente de farmacología clínica. $1^{\circ}$ edición. Barcelona. 2002, p. 398-9.

20. Micromedex. Drug-Reax. Greenwood Village, Colorado, Volumen 113, 2002.

21. Yen-Fu C, Avery AJ, Neil K, Johnson C, Dewey ME, Stockley IH Assessing de occurrence and preventability of prescribing potentially hazardous/contraindicated drug combinations in general (family) practice. Pharmacoepidemiol Drug Safety 2001; 10: S53.

22. Wrenger E, Muller R, Moesenthin M, Welte T, Frolich JC, Neumann $\mathrm{KH}$. Interaction of spironolactone with ACE inhibitors or angiotensin receptor blockers: analysis of 44 cases. BMJ 2003; 327: 147-9.

23. Durán Parrondo C, Rodríguez Moreno C, Tato Herrero F, Alonso Vence N, Lado Lado FL. Oral anticoagulation. An Med Interna (Madrid) 2003; 20: 377-84.

24. Mundet X, Artaza MA, Vidal X, Arnau JM. The use of digoxin in pri- mary care. Aten Primaria 1992; 9: 239-44.

25. Mundet X, Vidal X, Arnau JM. The characteristics of digoxin utilization in a population under emergency care for heart failure or auricular fibrillation. Med Clin 1993; 100: 94-7.

26. Rathore SS, Curtis JP, Wang Y, et al. Association of serum digoxin concentration and outcomes in patients with heart failure. JAMA 2003; 289: 871-8.

27. Fernando B, Savelyich BS, Avery AJ, Sheikh A, Bainbridge M, Horsfield $\mathrm{P}$, et al. Prescribing safety features of general practice computer systems: evaluation using simulated test cases. BMJ 2004; 328: 1171-2.

28. Magnus D, Rodgers S, Avery AJ. GPs' views on computerized drug interaction alerts: questionnaire survey. J Clin Pharm Ther; 27: 377-82.

29. Juurlink D, Muhammad M, Kopp A, Laupacis A, Redelmeier D. Drugdrug interactions among elderly patients hospitalised for drug toxicity. JAMA 2003; 289: 1652-58

30. Sakiara L, Nagib A. Risk of drug interaction: combination of antidepressants and other drugs. Rev Saúde Pública 2003; 37: 212-5

31. Tamblyn R, Huang A, Perreault R, Jacques A, Roy D, Hanley J, et al The medical office of the 21st century (MOXXI): effectiveness of computerized decision-making support in reducing inappropriate prescribing in primary care. CMAJ 2003; 169: 549-56.

32. Lazarou J, Pomeranz BH, Corey PN. Incidence of adverse drug reactions in hospitalized patients: a meta-analysis of prospective studies. JAMA 1998; 279: 1200-5.

33. Stockley I. Interacciones farmacológicas. $1^{\text {a }}$ edición. Barcelona: Pharma Editores S.L., 2003. 